\title{
The Design of Intelligent Cleaning Robot
}

\author{
Mi-mi Wang ${ }^{1, a, *}$, Ying Xin ${ }^{1, b}$, Haiyan Xin ${ }^{1, c}$ and Lizhen Zhang ${ }^{1, c}$ \\ ${ }^{1}$ Southeast University Chengxian College, Dongda Road No. 6, Nanjing, China \\ a yimi0516@163.com, b516273618@qq.com, ${ }^{\text {c } 983511889 @ q q . o c m, ~}$ \\ *corresponding author
}

Keywords: Intelligent cleaning robot; Path planning; Intelligent obstacle avoidance; prevent falling.

\begin{abstract}
The purpose of this thesis is to design and produce a low cost, low energy consumption, easy to use, suitable for family life, can realize the automatic cleaning of the intelligent cleaning robot. This design adopts the structured design principle, the design of intelligent cleaning robot based on MSP430 microcontroller is divided into 6 modules: cleaning function module, motion control module, human-computer interaction module, security detection module, route planning module, power supply module. This design uses one button operation method, through the infrared sensor intelligent obstacle avoidance, the installation of bilateral brush and vacuum cleaners cleaning. After experiment, it is proved that the design is reasonable and feasible.
\end{abstract}

\section{Introduction}

Early cleaning robots are mostly sold in Europe and the United States, the market of the right service robot is only beginning to take shape from about 2005 in china. With the improvement of domestic living standards, it gradually entered China. Many universities in the country have done a lot of research on clean robots and achieved some results, especially in the robot movement planning and control, which laid the foundation for the research and development of cleaning robot $^{[1]}$. Foreign intelligent cleaning robot compared to domestic, better performance, and many have a high degree of intelligent design, but the price is generally high, which makes foreign products can not meet the needs of more Chinese ordinary family. The purpose of this paper is to design and produce a low-cost, low-power, easy to use, suitable for family life, to achieve automatic cleaning of intelligent cleaning robot.

\section{The Design Strategy}

The design uses the principle of structural design, intelligent cleaning robot design is divided into five modules: cleaning function module, motion control function module, man-machine interaction module, safety detection module, route planning module, power supply module. To single-chip as the core, according to the smart cleaning robot design requirements, the use of various types of sensors for testing, to achieve the control of the functional modules to complete the design of the intelligent cleaning robot. The system function structure is shown in Figure 1. 


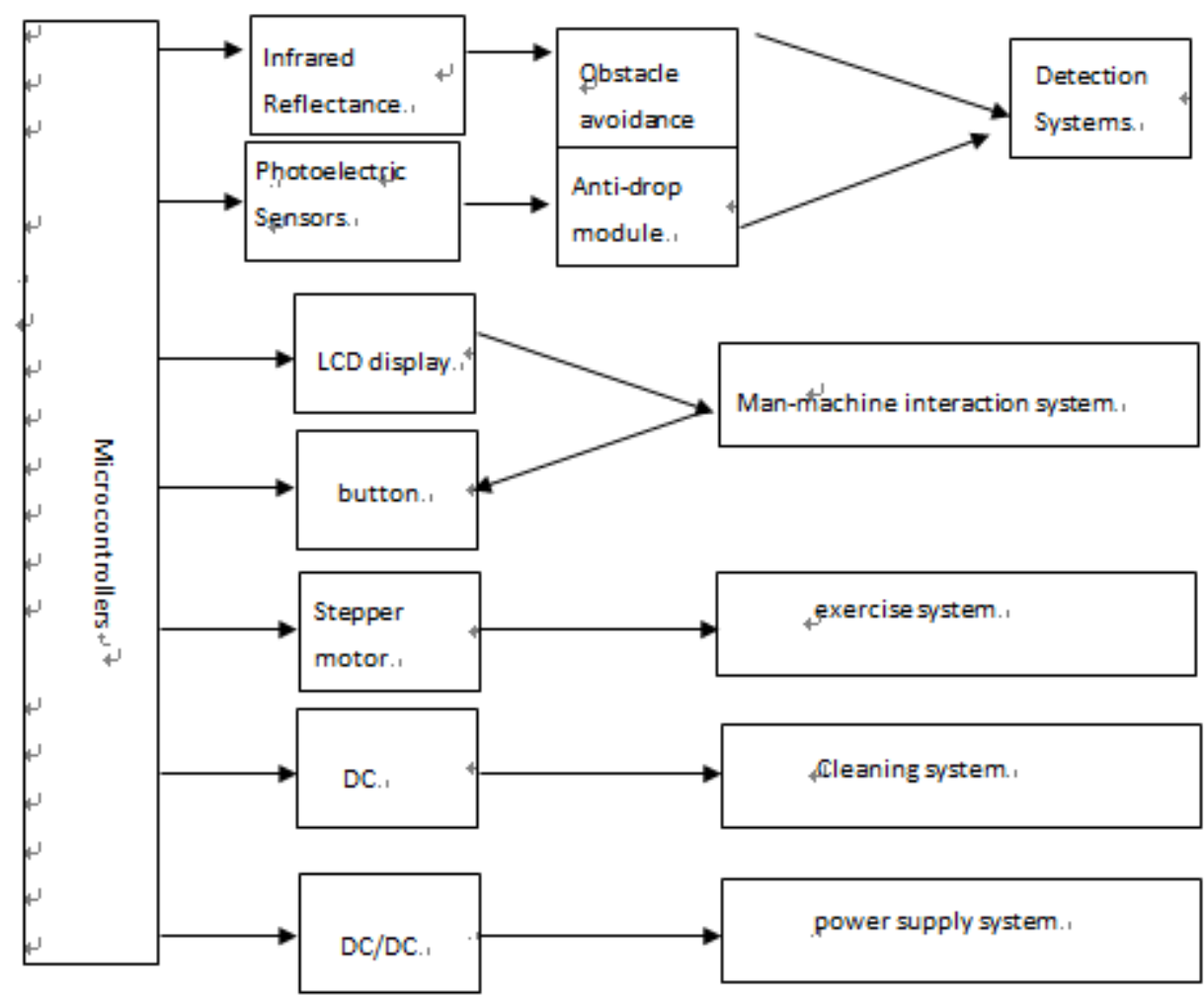

Figure1 The system function structure

\section{Hardware Design of The System}

\subsection{Overall Structural Design}

The system uses 16-bit low-power microcontroller MSP430F149 as the core controller, combined with sensors, motion modules, cleaning components module, to achieve intelligent control of cleaning robot. According to the functional requirements of the system, and the needs of the components analysis[2], start the distribution design of structural module. The distribution design of structural module is shown in Figure 2.

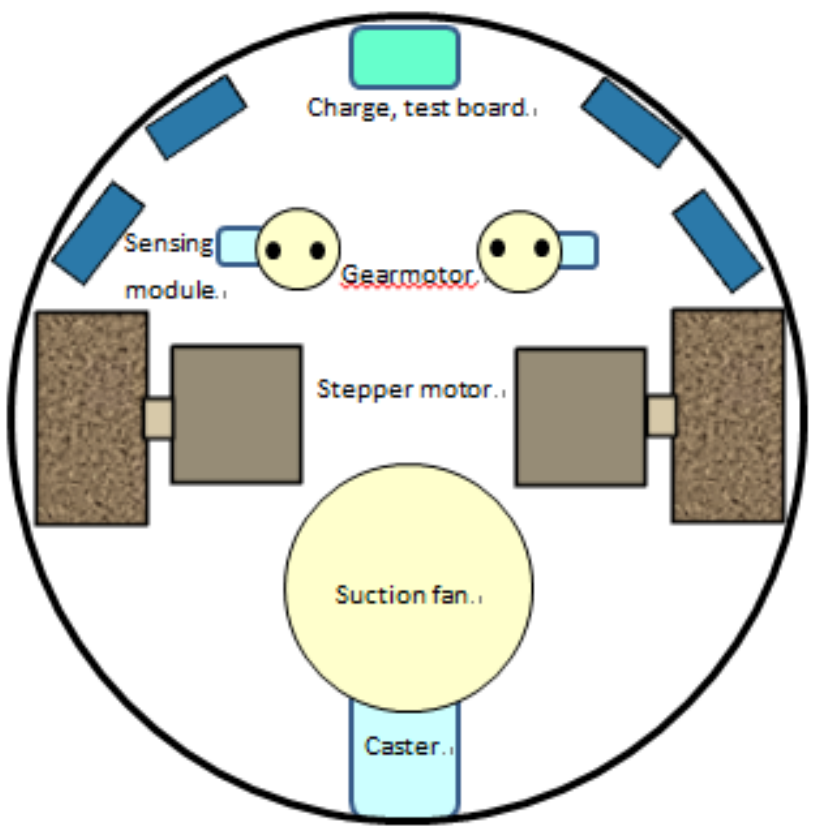

Figure2 The distribution design of structural module 


\subsection{Hardware Circuit Design}

In view of the functional analysis of the system, the need to complete the circuit design of the cleaning function module, the motion control function module, the human-computer interaction module, the safety detection module c, the route planning module and the power supply module ${ }^{[3]}$. Due to limited space, take the motion module as an example.

\subsubsection{Selection of Motor Module}

The motion module is mainly used for the load drag and direction control of the circuit. The stepper motor can directly convert the electrical pulse signal to the corresponding angular displacement or linear displacement without transformation. Therefore, in the digital control system control, the choice of stepper motor as a servo element is more suitable.

\subsubsection{Motion Module Circuit Design}

In the design, On both sides of the robot are installed stepper motor control of the rubber wheel, to achieve the load drag and direction control. Single-chip sent pulse signal to achieve the eight-step stepper motor power mode.

Through the ULN2003 chip on the signal drive amplification, stepper motor control requirements from the microcontroller so as to accurately achieve a variety of planning movement routes. The motion module design schematic is shown in Figure 3.

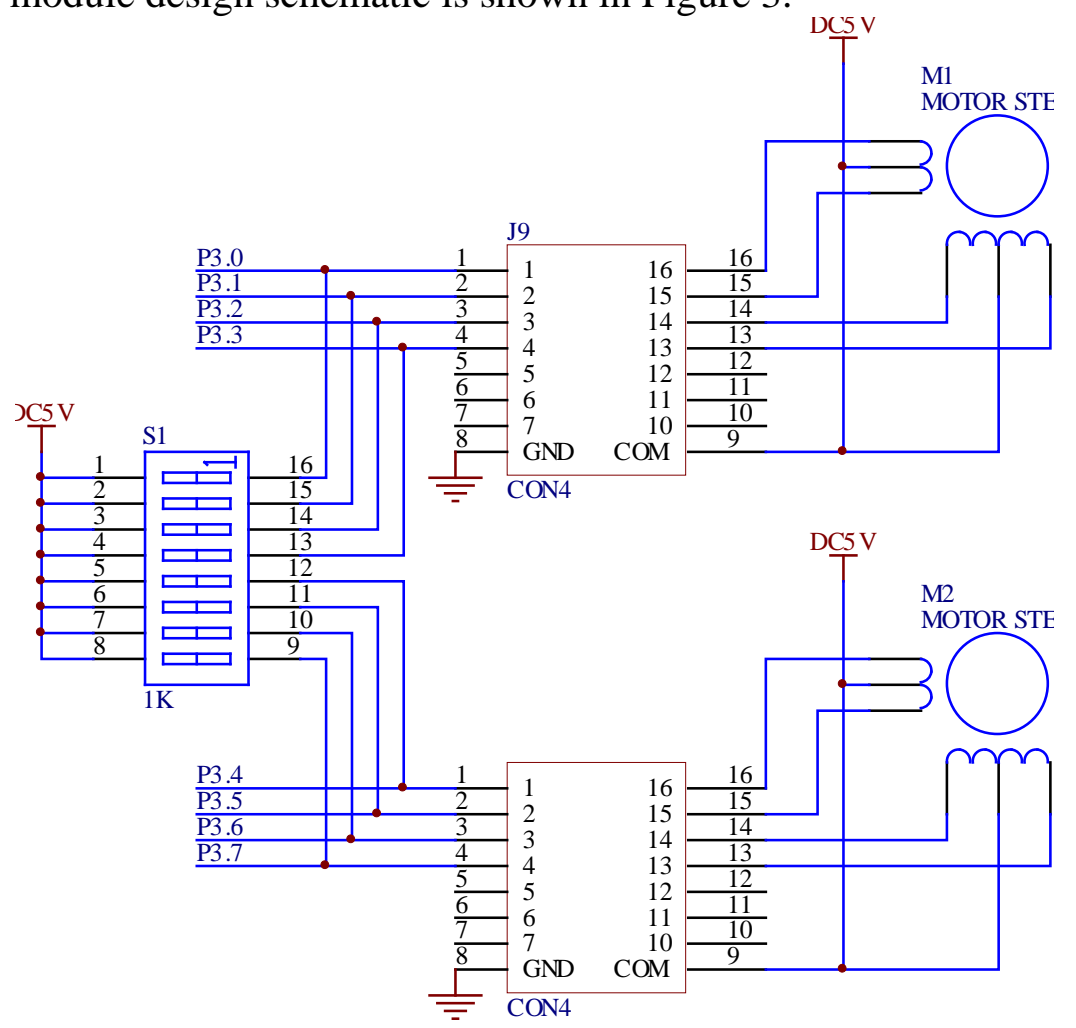

Figure3 The motion module design

\section{Software Design of The System}

The IAR Embedded Workbench, developed by IAR Systems in Sweden, is an integrated development environment that supports ARM, AVR, MSP430 and other chip core platforms and on a wide range of operating platforms. The design uses IAR Embedded Workbench as a software development platform, an uses $C$ language to program.

$\mathrm{C}$ language is one of the most widely used general computer programming language, is a highlevel language. C language compiler is simple, flexible and convenient, with a wealth of operators, data types. C language portability is good, with a program can be almost no modification, it applies to different single-chip platform, strong expression, the program readability is better. And it can run 
without the need for any operating environment support. C language is a structured language, with advantages of clear level, readability, please facilitate debugging and maintenance. $C$ language has a wealth of operators and data types, and a variety of complex data structure operations can be achieved. More prominently, the $\mathrm{C}$ language is suitable for a variety of models and operating systems.

According to the characteristics of the sensor and the control requirements, the program is divided into the program design of the route planning module, the programming of the manmachine interaction module, the program design of the motion module and the design of the cleaning module. When the system is running, execute the main program and complete the corresponding function. After the choice of working mode is completed, the system enter the different route planning state and start cleaning. At the same time, the system open the security detection module to complete the avoidance of obstacles, prevent the robot and other functions ${ }^{[4]}$.

\section{System Operation Results Analysis}

After the hardware circuit design, software programming, and the overall assembly and debugging, the intelligent cleaning robot has four route planning modes ${ }^{[5]}$, which include along the edge cleaning mode, automatic cleaning mode, random cleaning mode, intelligent cleaning robot cleaning mode. When the edge cleaning mode is selected, the smart cleaning robot is placed near the wall and the cleaning effect is observed by changing the angle between the initial position of the robot and the wall. The cleaning results are shown in Table 1.

Table 1 along the edge cleaning mode

\begin{tabular}{|c|c|}
\hline The angle intelligent cleaning robot and walls & Cleaning effect \\
\hline $\begin{array}{l}\text { between the front side of the robot and the wall } \\
\qquad 30^{\circ}\end{array}$ & Can be better to achieve along the edge cleaning \\
\hline between the left side of the robot and the wall $45^{\circ}$ & $\begin{array}{l}\text { Could achieve along the edge of the sweep, but at the } \\
\text { corner, about } 5 \mathrm{~cm} \text { from the corner area cannot be swept }\end{array}$ \\
\hline $\begin{array}{l}\text { between the front face of the robot and the wall } \\
\qquad 90^{\circ}\end{array}$ & Can be better to achieve along the edge cleaning \\
\hline between the left side of the robot and the wall $75^{\circ}$ & $\begin{array}{l}\text { Could achieve along the edge of the sweep, but at the } \\
\text { corner, about } 5 \mathrm{~cm} \text { from the corner area cannot be swept }\end{array}$ \\
\hline between the left side of the robot and the wall $60^{\circ}$ & Can be better to achieve along the edge cleaning \\
\hline $\begin{array}{l}\text { back to the wall at any angle (the left side is } \\
\text { closer to the wall) }\end{array}$ & $\begin{array}{l}\text { only straight, not along the edge, until Encounter the } \\
\text { next corner }\end{array}$ \\
\hline $\begin{array}{l}\text { back to the wall at any angle (the right side is } \\
\text { closer to the wall) }\end{array}$ & $\begin{array}{l}\text { only straight, not along the edge, until Encounter the } \\
\text { next corner }\end{array}$ \\
\hline
\end{tabular}

According to the experimental results, when the intelligent cleaning robot facing the wall, can better complete the cleaning task, cleaning a larger range, and can avoid obstacles. Since the rotation angle in the program is set to 15 degrees, or a multiple of 15 degrees, when the robot faces toward the wall and is at a multiple of 15 degrees, it is better to work parallel to the wall, and there is no angle of confusion. If the angle between the robot and the wall is not a multiple of 15 degrees, the edge effect changes, but the impact is not be too great. At the same time, through the use of edge brush can also reduce of the cleaning blind spot of the robot at the corner.

When the intelligent cleaning robot starts working on the back of the wall, the robot remains straight because the four infrared obstruction sensors do not detect an obstacle. No matter how the initial angle of the robot, it will always be more and more away from the wall, until the next corner, then return to normal. In this way, before the corner, the deviation will produce a part of the corner cannot be cleaned to. It is could that add two infrared sensors to detect the current direction on the left and right sides of the robot, so that when the robot is back to the wall, it can be rotated in situ until the side edge.

Experiments show that in the four route planning mode, the system can automatically complete the cleaning work, and have a better anti-crash function. 


\section{Conclusions}

In the design , MSP430 microcontroller as the core, stepper motor drive the overall load, and a series of infrared sensors do the security testing. So the intelligent cleaning robot is a low-cost, low power consumption, easy to use, suitable for family , and could achieve automatic cleaning.

\section{References}

[1]Iwashita, S,Murase, Y,Yasukawa,et al. Developing a service robot[J]. Mechatronics and Automation International Conference.2005,2(2):1057-1062.

[2]MC9S12DG128 Device User Guide[M].Freescale Sem iconductor， Inc. 2007.

[3] R.Saravanan, S.Rama balan, C.Balamurugan. Evolutionary collision-free optimal trajectory planning for intelligent robots[J].The International Journal of Advanced Manufacturing Technology,2004,53(6):85 - 89.

[4]Do-Hyeon Kim, Kwang-Baek Kim,Eui-Young Cha. Fuzzy truck control scheme for obstacle avoidance[J].Neural Computing and Applications.2009,18(7):801-811.

[5]Jinyan Shao, Guangming Xie,Long Wang, et al. Obstacle Avoidance and Path Planning Based on Flow Field for Biomimetic Robotic Fish[J]. Advances in Artificial Intelligence.2005,38(9):857860. 